\title{
Präklinische Versorgung von Extremitätenfrakturen und Luxationen
}

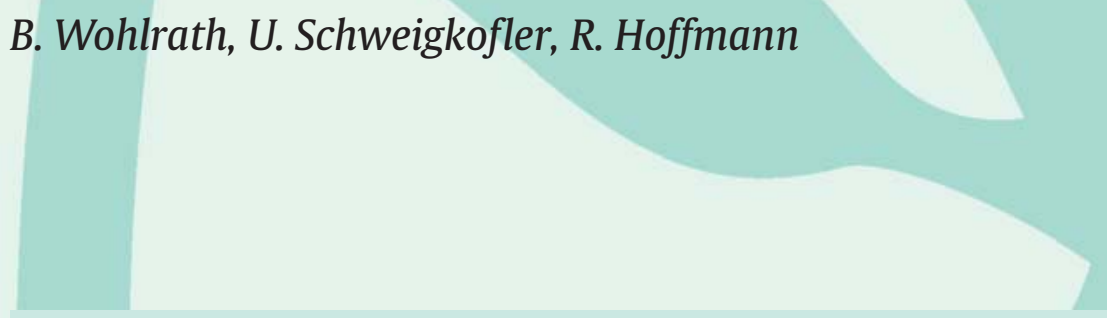

In diesem Übersichtsartikel werden die Relevanz der präklinischen Versorgung von Extremitätenfrakturen sowie die wichtigsten notärztlichen Maßnahmen im Management dieser Verletzungen dargestellt und Möglichkeiten gezeigt, sowohl offene als auch geschlossene Frakturen und Luxationen der Extremitäten präklinisch sicher zu versorgen.

\section{Einleitung}

Im Rahmen der präklinischen Patientenversorgung werden Notärzte häufig mit Frakturen und Luxationen an den Extremitäten konfrontiert. Der Jahresbericht 2014 des TraumaRegisters der DGU beschreibt insgesamt 42954 registrierte Patienten mit einem Injury Severity Score (ISS) von mindestens 16 in den Jahren 2011 -2013. Hiervon hatten $29,8 \%$ relevante Verletzungen der Extremitäten (AIS [Abbreviated Injury Scale] $\geq 3)[1]$.

Frakturen und Luxationen der Extremitäten können bei fast jedem Unfallmechanismus auftreten, sodass sie im Rettungsdienst häufig als isolierte Verletzung erscheinen. In der Gesundheitsberichterstattung des Bundes sind die Verletzungshäufigkeiten dokumentiert. So

\section{Abkürzungen}
AIS Abbreviated Injury Scale
ATLS Advanced Trauma Life Support
DGU Deutsche Gesellschaft für Unfallchirurgie
DMS Durchblutung, Motorik und Sensibilität
ISS Injury Severity Score
pDMS periphere Durchblutung, Motorik und Sensibilität
PHTLS Prehospital Trauma Life Support
RTW Rettungstransportwagen

zeigt sich z. B. in den Jahren 2000-2012 in Deutschland eine Häufigkeit von 165 Unterschenkelfrakturen oder 203 Femurfrakturen pro 100000 Einwohner [2]. Viele dieser Verletzungen werden präklinisch ohne Beteiligung von Notärzten behandelt. Besonders im Fall von eindrucksvollen offenen Verletzungen mit Blutungen oder deutlichen Dislokationen und damit verbundenen starken Schmerzen besteht eine Notarztindikation.

Auch wenn isolierte Extremitätenverletzungen nur selten eine akut lebensbedrohliche Situation darstellen, ist bereits die präklinische Versorgung äußerst wichtig für den Erfolg der späteren Rehabilitation des Patienten. Eine adäquate Therapie kann neben der Schmerzreduktion schon an der Unfallstelle helfen, Folgeschäden wie Weichteilquetschungen durch Knochenfragmentdruck, Infektionen oder Durchblutungsstörungen zu vermeiden, und sollte deshalb von jedem Notarzt beherrscht werden.

\section{Grundlagen}

\section{Erstuntersuchung}

Frakturen und Luxationen an den Extremitäten bieten häufig ein sehr eindrucksvolles Bild, zumal sie meistens mit starken Schmerzen einhergehen. Allerdings darf man sich bei der Erstuntersuchung des Patienten davon nicht beeindrucken oder ablenken lassen. 
Bei der Erstuntersuchung können standardisierte prioritätenorientierte Algorithmen wie PHTLS (Prehospital Trauma Life Support) helfen. Lebensrettende Sofortmaßnahmen stehen im Vordergrund. In diesen Situationen wird auch eine offensichtliche Fraktur oder Luxation an den Extremitäten noch nicht primär reponiert. Eine offensichtlich frakturassoziierte, starke Blutung nach außen mit Kreislaufrelevanz muss allerdings sofort gestillt werden, bevor die initiale Beurteilung des Patienten fortgesetzt wird $[3,4,20]$.

Starke Blutungen, die die Kreislauffunktion beeinträchtigen können, müssen sofort gestillt werden!

\section{Primary Survey}

Anschließend beginnt man mit der strukturierten Untersuchung des Patienten nach dem ABCDE-Schema, dem „Primary Survey“ (Tab. 1). Es wird eine prioritätenorientierte Untersuchung des Verletzten durchgeführt, und bei Auftreten eines Problems wird dieses sofort behoben, bevor man zum nächsten Punkt

\section{Tabelle 1}

Primary Survey nach ATLS und PHTLS.

\begin{tabular}{|ll|}
\hline A Airway and C-Spine Protection & Atemweg sichern und HWS-Stabilisierung \\
\hline B Breathing & Beatmung und Ventilation \\
\hline C Circulation & Kreislauf \\
\hline D Disability & neurologischer Status \\
\hline E Exposure/Environment & Entkleiden und Wärmeerhalt \\
\hline
\end{tabular}

Tabelle 2

Zu erwartender innerer Blutverlust bei geschlossenen Frakturen [5].

\begin{tabular}{|l|l|}
\hline geschlossene Extremitätenfraktur & zu erwartender Blutverlust \\
\hline Radius/Ulna & $50-400 \mathrm{ml}$ \\
\hline Humerus & $100-800 \mathrm{ml}$ \\
\hline Tibia & $100-1000 \mathrm{ml}$ \\
\hline Femur & $300-2000 \mathrm{ml}$ \\
\hline Becken & $500-5000 \mathrm{ml}$ \\
\hline
\end{tabular}

weitergeht. Somit können akut lebensbedrohliche Zustände schnell erkannt und behoben werden.

Um die Behandlung lebensbedrohlicher Verletzungen nicht zu verzögern, werden Extremitätenfrakturen oder -luxationen in diesem Stadium noch nicht behandelt, es sei denn, es besteht aufgrund einer starken Blutung eine Kreislaufrelevanz. Das Bedrohungspotenzial dieser Blutungen muss in der initialen Gesamtbeurteilung des Patienten daher rasch eingeschätzt werden (Tab. 2).

Cave. Die Behandlung lebensbedrohlicher Verletzungen und der Transport in eine geeignete Zielklinik in solchen Situationen dürfen durch die Versorgung von nicht lebensbedrohlichen Verletzungen nicht verzögert werden.

\section{Secondary Survey}

Nachdem die akut lebensbedrohlichen Probleme ausgeschlossen oder behandelt sind, wird der Verletzte im Rahmen des „Secondary Survey“ von Kopf bis Fuß untersucht und eine Anamnese erhoben. Diese Untersuchung darf besonders bei einem schweren Trauma mit denkbar schweren bis lebensbedrohlichen Verletzungen den Abtransport in die geeignete Zielklinik nicht verzögern. Im Rahmen des Secondary Survey werden nun auch Extremitätenverletzungen untersucht und behandelt, die nicht unter den Punkt „C“ (Blutung/Kreislauf) des Primary Survey fallen $[3,4]$.

\section{Klassifikation}

Man unterscheidet präklinisch zwischen

- offenen Frakturen und

- geschlossenen Frakturen.

Die offenen Frakturen, bei denen der schützende Weichteilmantel penetriert ist, sind auf den ersten Blick deutlich eindrucksvoller als die geschlossenen Frakturen (Abb. 1 u. Abb. 2). Jedoch können auch bei geschlossenen Frakturen erhebliche Weichteilschäden, die sogar extremitätengefährdend sein können, vorliegen.

Die Klassifikationen nach Tscherne und Oestern sowie Gustilo und Anderson geben einen Überblick über die Schweregrade der Weichteilschädigung bei offenen (O) und geschlossenen Frakturen $(G)$ und sollten vom Notarzt „grob eingeschätzt“ und entsprechend dokumen- 


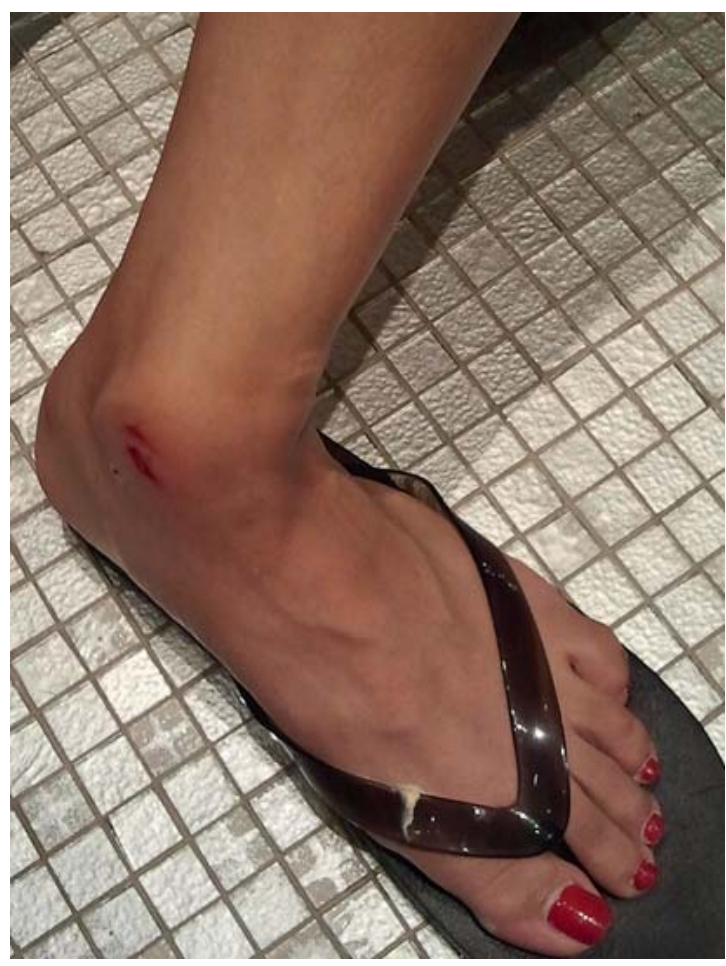

Abb. 1 Geschlossene (G1) Sprunggelenkluxationsfraktur.

\section{Tabelle 3}

Klassifikation des Weichteilschadens geschlossener Frakturen nach Tscherne und Oestern [6].

\begin{tabular}{|c|c|}
\hline Grad & Kennzeichen \\
\hline Grad 0 & - fehlende oder unbedeutende Weichteilverletzung \\
\hline Grad 1 & $\begin{array}{l}\text { - oberflächliche Schürfung oder Kontusion durch } \\
\text { Fragmentdruck von innen }\end{array}$ \\
\hline Grad 2 & - tiefe kontaminierte Schürfung sowie Haut- oder \\
& Muskelkontusion \\
\hline Grad 3 & $\begin{array}{l}\text { ausgedehnte Hautkontusion, -quetschung oder } \\
\text { Zerstörung der Muskulatur }\end{array}$ \\
- & subkutanes Décollement \\
- manifestes Kompartment-Syndrom
\end{tabular}

tiert werden. Eine definitive Klassifikation des Weichteilschadens ist in der Regel erst intraoperativ in der Klinik und/oder im Verlauf möglich (Tab. 3 u. Tab. 4) $[5,6]$.

Eine Einteilung der Frakturen nach Dislokationsgrad ist präklinisch nicht möglich. Es ist lediglich eine Aussage über eine nach außen sichtbare Fehlstellung der Extre-

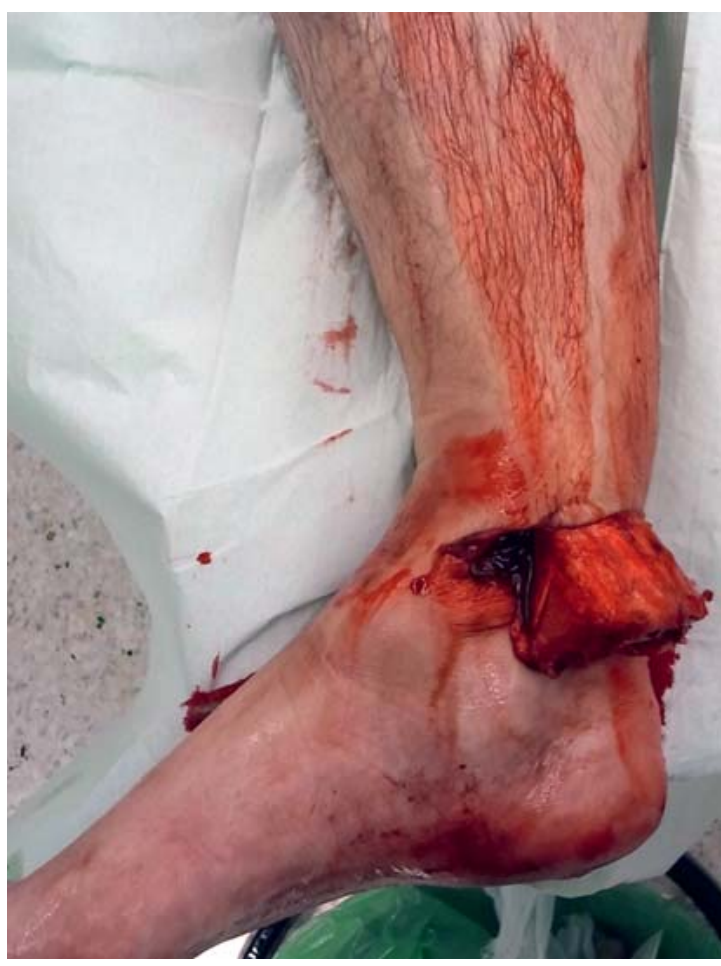

Abb. 2 Offene (03) Sprunggelenkluxationsfraktur.

\section{Tabelle 4}

Klassifikation der offenen Frakturen nach Gustilo und Anderson [7].

\begin{tabular}{|l|l|}
\hline Grad & Kennzeichen \\
\hline Grad 1 & - Hautläsion $<1 \mathrm{~cm}$ \\
& - Durchtrennung der Haut \\
& - Fragmentdurchspießung von innen \\
\hline Grad 2 & - Hautläsion $>1 \mathrm{~cm}$ \\
& - Fragmentdurchspießung von außen durch \\
& direktes Trauma \\
\hline Grad 3 & - ausgedehnter Weichteilschaden \\
& A: mit noch adäquater Knochendeckung \\
& B: Deperiostierung und freiliegender Knochen \\
& C: rekonstruktionspflichtige arterielle Gefäßversorgung
\end{tabular}

mität möglich, die sich in der Länge, Achse oder Rotation zeigen kann. Die Umstände des Unfalls können Hinweise auf die Schwere der Verletzung geben. Insbesondere sind hierbei zu beurteilen:

- der Unfallmechanismus,

- die kinetische Energie der Schadenseinwirkung und

- begleitende Sach- oder Personenschäden. 
Sowohl die initiale Einschätzung des Weichteilschadens bei offenen und geschlossenen Frakturen als auch die Fehlstellung der Extremität müssen vom Notarzt dokumentiert und an die Kollegen in der Klinik weitergegeben werden.

\section{Präklinische Diagnostik}

\section{Anamnese}

Soweit es die Zeit zulässt, empfiehlt es sich, die Unfallstelle genau in Augenschein zu nehmen und sich den Unfallhergang sowie die Unfallkinematik klar zu machen. Eine möglichst genaue Schmerzangabe durch den Patienten erleichtert die Suche nach Verletzungen. Jedoch ist zu beachten, dass starke Schmerzen an einer Stelle von Verletzungen, die anderweitig vorliegen und möglicherweise nicht so schmerzhaft sind, ablenken können. Stresshormone können den Schmerz initial teilweise oder sogar ganz unterdrücken (Beispiel: Schmerzfreiheit bei manchen Amputationsverletzungen).

Cave. Starke Schmerzen an einer Stelle können von anderen, vielleicht schwerwiegenderen Verletzungen ablenken, die dann übersehen werden.

\section{Inspektion}

Die Inspektion während des Second Survey ist unter „optimierten“ Bedingungen mit ausreichender Beleuchtung durchzuführen. Meist ist es dafür notwendig, den Patienten zu entkleiden, wobei hier streng auf den Wärmeerhalt geachtet werden muss (RTW). Die Entkleidung muss schonend und schmerzfrei erfolgen und die Verletzungsregionen müssen voll zugänglich gemacht werden. Hierzu muss die Kleidung regelhaft aufgeschnitten werden. Um eine möglichst detailgetreue Beschreibung des gesehenen Befundes an das Klinikteam weitergeben zu können, ist ein Foto vor der Reposition oder Verbandanlage nützlich, wobei auf den Datenschutz und die Persönlichkeitsrechte des Patienten $\mathrm{zu}$ achten ist [8].

Ein Bild sagt mehr als tausend Worte.

\section{Palpation und Funktionstest}

Durch das Abtasten der gesamten Extremität können besonders beim bewusstlosen Patienten geschlossene Verletzungen erkannt werden. Hierbei ist besonders auf die sicheren und unsicheren Frakturzeichen zu achten (Tab. 5).

\section{Praxis}

\section{Schmerzen vermeiden}

Sobald man ein sicheres Frakturzeichen gefunden hat, sollte man die Suche nach weiteren Frakturzeichen des betroffenen Knochens abbrechen, um unnötige Schmerzen zu vermeiden.

\begin{tabular}{|l|l|}
\hline Tabelle $\mathbf{5}$ \\
\hline Sichere und unsichere Frakturzeichen. \\
\hline sichere Frakturzeichen & unsichere Frakturzeichen \\
\hline abnorme Fehlstellung & Schwellung, Konturdeformität \\
\hline sichtbare Knochenfragmente & freiliegender Knochen \\
\hline Krepitation & Schmerz \\
\hline abnorme Beweglichkeit & Funktionseinschränkung \\
\hline
\end{tabular}

Soweit schmerzbedingt möglich, sind alle Gelenke kurz auf ihre Beweglichkeit und Stabilität hin zu prüfen. Wenn möglich, sollte der wache Patient auch aufgefordert werden, die Extremität aktiv zu bewegen. Alle Extremitäten müssen im Seitenvergleich untersucht werden [8-10].

\section{Neurologie und Durchblutung}

Bei jedem Verdacht auf eine Extremitätenverletzung ist eine erste Prüfung der peripheren Durchblutung, Motorik und Sensibilität (pDMS) erforderlich. Die Ergebnisse müssen notiert und in der Klinik verifiziert werden. Durch orientierendes Ertasten der peripheren Pulse und der einfachen Testung der Sensibilität durch Abstreichen der Extremität sowie aktives Bewegen der Extremität durch den Verletzten kann dies schnell und einfach dokumentiert werden. Auch diese Untersuchungen sind immer im Seitenvergleich durchzuführen $[8,9]$. 


\section{Präklinische Therapie}

\section{Geschlossene, nicht dislozierte Frakturen}

Jede Fraktur sollte, wenn möglich, vor dem Transport oder vor Bewegung des Patienten ruhiggestellt werden. Hierfür ist gegebenenfalls eine Schmerztherapie notwendig.

Schon der Verdacht auf eine Fraktur genügt, um eine Schienung zu initiieren. Hierzu sollten mindestens das jeweils proximal und distal von der Fraktur gelegene Gelenk mit in die Immobilisation einbezogen werden. Die Extremität sollte dann flach gelagert werden. Eine adäquate Schienung dient nicht nur zur Schmerztherapie, sondern kann auch das Ausmaß der Schwellung eindämmen und Fettembolien sowie weitere Weichteilschädigungen, Blutungen oder neurologische Schäden verhindern.

Besteht der Verdacht auf eine Fraktur, sollte die betroffene Extremität vor dem Transport oder Bewegungen geschient werden.

Eine zusätzliche Kühlung kann ebenfalls Schwellungen und Schmerzen reduzieren, jedoch ist dabei eine Hypothermie des Patienten unbedingt zu vermeiden. Gegenstände, die möglicherweise die Extremität oder Teile davon abschnüren könnten, wie z.B. Ketten und Ringe, sollten möglichst frühzeitig abgenommen werden $[11,12]$.

\section{Dislozierte Frakturen}

Die grundsätzliche Therapie bei dislozierten Frakturen entspricht den oben beschriebenen Maßnahmen bei nicht dislozierten Frakturen. Jedoch sollte man bei sichtbar dislozierten Frakturen besonderes Augenmerk auf die periphere Durchblutung, Motorik und Sensibilität legen.

Grob dislozierte Frakturen sollten vor dem Transport möglichst reponiert werden, um die umgebenden Weichteile vor zusätzlichen Druckschäden von innen durch Knochenteile zu bewahren. Das Ziel der Reposition ist nicht die anatomische Stellung, viel mehr hat die Reposition zum Ziel:

- die achsgerechte Lagerung der Extremität,

- die Wiederherstellung der peripheren Durchblutung und

- die Druckentlastung der Weichteile.

\section{Praxis}

Schmerzen vermeiden

Eine Reposition ist sehr schmerzhaft! Beim wachen Patienten muss auf eine ausreichende Analgesie geachtet werden.

Hierfür reicht unter entsprechender Analgesie meist ein moderater axialer Zug an der Extremität aus, sodass diese dann in annähernder Neutralstellung fixiert und geschient werden kann. Bei der Reposition ist sowohl vor als auch nach der Durchführung die periphere Durchblutung, Motorik und Sensibilität zu testen und zu dokumentieren. Ein neurologisches oder vaskuläres Defizit distal der Fraktur erfordert einen sofortigen Repositionsversuch $[8,12,13]$.

Grob dislozierte Frakturen sollten, insbesondere bei begleitender peripherer Ischämie oder neurologischer Symptomatik, präklinisch reponiert bzw. in eine achsgerechte Stellung gebracht werden. Die periphere DMS muss vor und nach der Reposition dokumentiert werden.

\section{Offene Frakturen}

Bei offenen Frakturen steht neben der Blutungskontrolle und Weichteilentlastung die Vermeidung von Infektionen im Vordergrund. Besteht eine massive und potenziell lebensbedrohliche Blutung nach außen, so sollte diese bereits im Primary Survey angegangen werden. Entsprechend dem ABCDE-Schema [20] werden starke, spritzende und direkt lebensbedrohliche Blutungen mit höchster Priorität behandelt. Spätestens allerdings bei „C“ sollten größere Blutungen, die nicht unmittelbar lebensbedrohlich sind, erkannt und behandelt werden. Kleinere Blutungen werden im Rahmen des Secondary Survey angegangen $[3,8,12]$. Zur Blutungskontrolle an den Extremitäten kann ein Stufenschema angewendet werden (Abb. 3).

Liegt bei einer offenen Fraktur keine lebensbedrohliche Blutung vor oder ist diese bereits gestillt, dann sollte der Fokus auf die Infektvermeidung gelegt werden. Hierfür werden alle offenen Wunden sofort von grobem Schmutz und Fremdkörpern befreit und mit einer sterilen Wundabdeckung versorgt. Die Wunden werden präklinisch nicht mit desinfizierenden Lösungen behandelt $[3,8,12,15]$. 
Stufenschema zur Blutstillung aktiver Blutungen nach außen

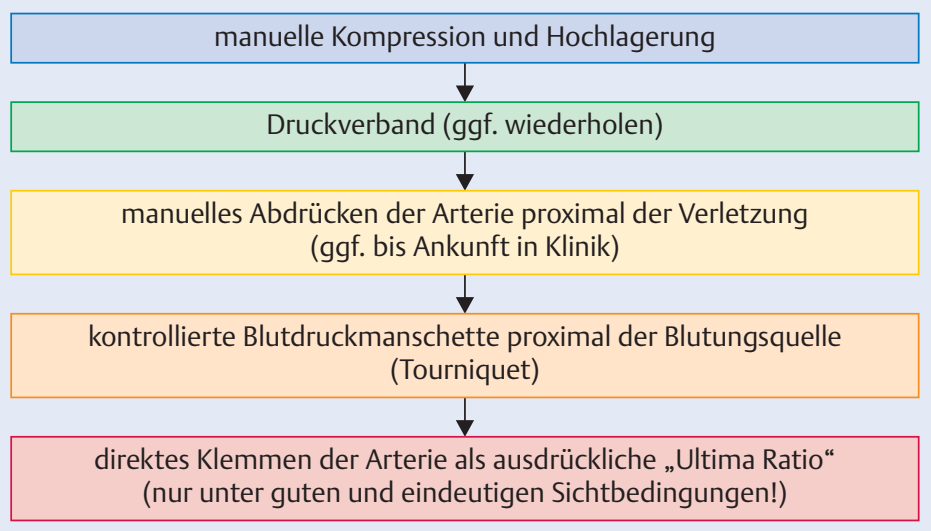

Abb. 3 Stufenschema zur Blutstillung aktiver Blutungen nach außen.

Danach sollten offene Verletzung genau wie geschlossene Verletzungen durch moderaten Längszug reponiert, immobilisiert und flach gelagert werden. Eine potenzielle „Keimverschleppung“ nach innen ist zu diesem Zeitpunkt vernachlässigbar. Die angelegten sterilen Verbände sollten erst im OP wieder geöffnet werden $[12,15]$. Auch hier kann es wieder nützlich sein, den Befund vor der Verbandanlage in einem Bild zu dokumentieren, um den aufnehmenden Kollegen in der Klinik eine möglichst detailgetreue Übergabe liefern zu können.

Besonders bei verlängerter Rettungszeit kann eine präklinische Antibiotikagabe in Erwägung gezogen werden, wenn diese mitgeführt wird, da sich nach 5 Stunden die Infektionsgefahr deutlich erhöht. Als Antibiotikum wählt man in der Regel ein Cephalosporin der 2. Generation $[15,18,19]$. In Deutschland spielt dies allerdings aufgrund der moderaten Rettungszeiten keine Rolle, ein Antibiotikum wird in aller Regel nicht mitgeführt.

Bei offenen Frakturen stehen die Blutungskontrolle und die Vermeidung von Infektionen im Vordergrund.

\section{Amputationen}

Bei Amputationsverletzungen an den Extremitäten ist zusätzlich zur Blutungskontrolle und Infektvermeidung auf das Amputat zu achten. Neben der Blutstillung und der Säuberung des Stumpfes von grober Verschmutzung sowie dem Anlegen eines sterilen
Verbandes muss das Amputat geborgen werden, wobei möglichst alle Anteile direkt mitgenommen oder beim zeitkritischen Patienten gegebenenfalls nachgebracht werden sollten.

Das Amputat wird nach der Säuberung von grober Verschmutzung in sterile, feuchte Kompressen gewickelt und in einen sterilen Amputatbeutel gelegt. Diesen Beutel legt man, nachdem er verschlossen wurde, in einen weiteren, mit Eiswasser gefüllten Beutel, um eine indirekte Kühlung zu erreichen und um Kälteschäden am Amputat zu verhindern.

Das Amputat muss steril verpackt und gekühlt mitgenommen werden.

Die Lokalisation und das Ausmaß der Amputation ist bei der Auswahl der Zielklinik zu beachten und sollte vorher genau angekündigt werden $[12,16]$.

\section{Blutungskontrolle}

Extremitätenfrakturen sind nicht selten mit starken Blutungen verbunden, die sowohl nach innen als auch nach außen auftreten können. Besonders die Blutungen nach innen sind oft schwer zu erkennen. Eine lebensbedrohliche innere Blutung bei Extremitätenverletzungen tritt in der Regel aber nur am Oberschenkel auf, die sich meist durch eine Reposition bzw. achsgerechte Lagerung schon etwas eindämmen lässt $[11,12]$. Besonders bei proximalen Oberschenkelfrakturen sollte man als Differenzialdiagnose auch immer an die Beckenfraktur denken und gegebenenfalls das Becken, z.B. mithilfe eines Beckengurtes, von extern stabilisieren.

Bei Blutungen nach extern muss zwingend zwischen akut und unmittelbar lebensbedrohlichen Blutungen und nicht akut lebensbedrohlichen Blutungen unterschieden werden. Jede spritzende und stark blutende Wunde, die eine akute Bedrohung darstellt, muss sofort versorgt werden $[3,12]$.

Bei der Blutstillung geht man in mehreren Stufen vor (Abb. 3).

\section{Manuelle Kompression, Hochlagerung der Extre-} mität. An erster Stelle stehen die manuelle Kompression der Wunde und gegebenenfalls die Hochlagerung der Extremität. 
Druckverband. Im Folgenden kann ein Druckverband angelegt werden, bei dem sich ein noch geschlossenes Verbandspäckchen über der Wunde als gute Fokussierung des Druckes eignet (gegebenenfalls wiederholen).

Arterie manuell abdrücken. Sollte auch der zweite Druckverband keine ausreichende Blutstillung erzielen, so sollte versucht werden, die Arterie proximal der Wunde mit der Hand zu komprimieren.

Tourniquet. Wenn keine dieser Maßnahmen zur Blutstillung führt, kann über die Anlage eines Tourniquets nachgedacht werden, was jedoch aufgrund der damit verbundenen Gewebeschädigung sehr kritisch überlegt werden sollte. Als Ersatz für ein Tourniquet kann eine Blutdruckmanschette dienen.

Wichtig ist dabei, dass ausreichend Druck aufgebaut wird, der über dem arteriellen Blutdruck liegt, um neben den Venen auch die Arterien komplett zu komprimieren, da sonst die Blutung noch verschlimmert wird.

Eine entsprechende Analgesie und Dokumentation inklusive der Uhrzeit der Anlage sind hierbei zwingend erforderlich.

Abklemmen der Arterie. Von einer direkten Abklemmung des Gefäßes sollte man präklinisch absehen. Diese Maßnahme verbleibt als „Ultima Ratio“, wenn anders keine Blutstillung zu erreichen ist und ein Tourniquet nicht angelegt werden kann $[8,12,13,17]$. Da das Abklemmen einer Arterie unter Notfallbedingungen nur ungezielt und potenziell schadenvermehrend erfolgen kann, wird hiervon grundsätzlich abgeraten.

Neu entwickelte Hilfsmittel zur temporären Blutstillung, wie z.B. die IT-Clamp, sind in der Präklinik in Deutschland in aller Regel nicht verfügbar und sollten auch nur in ganz speziellen Fällen eingesetzt werden.

\section{Luxationen}

Gelenkluxationen sind fast immer mit massiven Schmerzen verbunden. Besonders unter diesem hohen Leidensdruck der Patienten stellt sich in der präklinischen Situation häufig die Frage, ob eine bestehende Luxation reponiert werden soll oder ein Transport im luxierten Zustand unter adäquater Analgesie durchgeführt wird. Hier wird uns in den Leitlinien leider kein allgemeingültiger Algorithmus vorgegeben. Somit ist die Entscheidung für oder gegen die Reposition häufig

\section{Praxis}

Repositionsversuch

Es gilt allerdings allgemein, dass bei deutlicher Luxation eines Gelenkes mit gestörter Durchblutung, Motorik und Sensibilität ein Repositionsversuch unter ausreichender Analgesie unternommen werden soll [8].

eine Einzelfallentscheidung, die nicht selten auch von der persönlichen Erfahrung des Notarztes abhängt.

Bei Luxationen von großen Gelenken, wie Hüfte, Knie oder Schulter, kann bei vollständig intakter Durchblutung, Motorik und Sensibilität ein Transport unter Analgesie ohne vorherigen Repositionsversuch in die Klinik erfolgen, wenn dies in einem vertretbaren Zeitrahmen möglich ist. Besonders an der Schulter ist eine Differenzierung zwischen Fraktur und Luxation schwer, sodass es bei zu brüsken Repositionsversuchen zu einer Dislokation der Kopfkalotte kommen kann.

Bei kleineren Gelenken, speziell beim Sprunggelenk, kommt es durch die Luxation, meist in Kombination mit einer Fraktur, jedoch häufig zu einer deutlichen Weichteilkompromittierung, sodass eine sofortige Reposition durch einfachen Längszug zum Schutz vor weiteren Schäden an Haut, Nerven und Gefäßen schon am Unfallort angezeigt ist $[10,12]$.

Um eine Patellaluxation zu reponieren, genügt es häufig schon, das Bein vollständig zu strecken, wenn notwendig wird dann noch ein sanfter seitlicher Druck auf die Patella ausgeübt.

Spätestens beim Eintreffen in der Klinik sollte allerdings eine Reposition von luxierten Gelenken zeitnah durch einen darin Erfahrenen nach erfolgter Röntgendiagnostik und in angepasster Analgesie/Anästhesie erfolgen. Zur Reposition genügt unter entsprechender Analgesie häufig ein konstanter und moderater axialer Zug distal der Luxation. Die Dokumentation der peripheren Durchblutung, Motorik und Sensibilität vor und nach der Reposition ist erforderlich.

Besteht der Verdacht auf eine Luxationsfraktur oder besteht eine Instabilität des Gelenkes, so muss die Extremität entsprechend der oben beschriebenen Frakturversorgung grob reponiert und geschient werden. Offene Verletzungen bei Luxationen werden wie bei den oben beschriebenen offenen Frakturen versorgt. 
Sprunggelenkluxationsfrakturen sollten schnellstmöglich durch einen darin Erfahrenen reponiert werden.

\section{Lagerungshilfen}

Die Auswahl der präklinisch mitgeführten Stabilisierungsmaterialien ist regional sehr unterschiedlich. Es kommen viele verschiedene kommerzielle Schienungsmaterialien zum Einsatz, mit denen sich jeder im Rettungsdienst Tätige in seiner jeweiligen Region vertraut machen sollte. Einige häufig eingesetzte Materialien werden im Folgenden beschrieben und in Tab. 6 als Übersicht dargestellt.

\section{Vakuummatratze}

In fast allen Rettungsdienstbereichen werden Vakuummatratzen eingesetzt. Die Vakuummatratze hat den Vorteil, dass man mit ihr mehrere Körperregionen gleichzeitig immobilisieren kann, und eignet sich deshalb besonders beim Mehrfachverletzten, bei dem auch eine Immobilisation des Rumpfes gewünscht ist. Besonders bei Frakturen der proximalen unteren Extremität (Oberschenkel, Hüfte) bietet die Vakuummatratze eine gute Stabilität. Sie kann jedoch bei Frakturen der Arme praktisch nicht eingesetzt werden, außerdem ist die Anmodulierung recht zeitintensiv.

\section{Luftkammerschienen}

Luftkammerschienen sind in unterschiedlichen Größen für Arme und Beine erhältlich. Sie können einfach und schnell angelegt werden und bieten eine gute Stabilität. Sie werden lediglich über die Extremität gezogen und dann aufgeblasen. Jedoch sind sie aufgrund des innen entstehenden Druckes ungeeignet für Frakturen mit größeren Weichteilschäden und können selbst auch sekundäre Weichteilschäden durch zu starkes Aufpumpen provozieren. Die Gefahr der iatrogenen Schädigung durch diese Schienen ist so groß, dass ihr Einsatz sehr kritisch überlegt werden sollte.

\section{Vakuumschienen}

Vakuumschienen sind ebenfalls in unterschiedlichen Größen für Arme und Beine erhältlich. Sie werden um die Extremität gelegt und mit Klettverschlüssen befestigt, danach wird die Luft darin abgesaugt. Sie bieten somit eine sehr gute Stabilität, können jedoch ebenfalls eine Kompression auf Haut und Fraktur ausüben und beim Absaugen einen Repositionsverlust hervorrufen.

\section{Tabelle 6}

Übersicht präklinisch häufig genutzter Schienungsmaterialien.

\begin{tabular}{|c|c|c|}
\hline Material & Vorteile & Nachteile \\
\hline Vakuummatratze & $\begin{array}{l}\text { - mehrere Körperregionen gleichzeitig } \\
\text { - gute Stabilität }\end{array}$ & $\begin{array}{l}\text { - hoher Zeitaufwand } \\
\text { - ungeeignet für die Arme }\end{array}$ \\
\hline Luftkammerschiene & $\begin{array}{l}\text { - schnell angelegt } \\
\text { - gute Stabilität }\end{array}$ & - potenziell sekundärer Weichteilschaden bei Überinsufflation \\
\hline Vakuumschiene & - sehr gute Stabilität & $\begin{array}{l}\text { - potenziell Kompression auf Fraktur und Weichteile } \\
\text { - Stellungsverlust }\end{array}$ \\
\hline Kunststoffsplint & - sehr schnell angelegt & - geringe Stabilität \\
\hline Alupolsterschiene & $\begin{array}{l}\text { - flexibel } \\
\text { - leicht }\end{array}$ & $\begin{array}{l}\text { geringe Stabilität } \\
\text { - leichte Verbiegbarkeit }\end{array}$ \\
\hline Dreiecktuch & - schnell angelegt & $\begin{array}{l}\text { - mangelnde Stabilität } \\
\text { - nur für Schulter und Arm geeignet }\end{array}$ \\
\hline
\end{tabular}




\section{Einfache Kunststoffsplints (z. B. Prosplint-Schienen)}

Kunststoffsplints in verschiedenen Größen für Arme und Beine werden unter die Extremität gelegt und dann darüber mit Klettverschlüssen verschlossen. Sie können sehr schnell und ohne Hilfsmittel angelegt werden, bieten jedoch nur eine geringe Stabilität (Abb. 4).

\section{Alupolsterschiene}

Als Nachfolgermodell der Cramer-Schiene kann die Alupolsterschiene durch ihre flexible Form an jede Extremität angepasst werden und ist dabei sehr leicht. Sie wird in der eigenen Hand vorgeformt, an die verletzte Extremität angelegt und mit Binden daran fixiert. Schon bei der Formung sollte an die korrekte Stellung der Fraktur und der zu schienenden Gelenke gedacht werden. Die großen Nachteile dieser Schienen sind die geringe Stabilität und die Verbiegbarkeit. Sie wird deshalb hauptsächlich am Unterarm benutzt (Abb. 5).

\section{Dreiecktuch}

Zur Stabilisierung der Schulter und des Armes eignet sich das Dreiecktuch, welches um den Unterarm und den Hals gelegt wird, um den Arm in einer Beugung von $90^{\circ}$ im Ellenbogen nahe am Oberkörper zu halten. Es bietet darüber hinaus allerdings keine Stabilität. An der unteren Extremität kann es zum Zusammenbinden der Unter- oder Oberschenkel benutzt werden, um die gesunde Seite als Gegenschienung zu verwenden.

Bei jeder Schienung ist vor und nach Anlage der Lagerungshilfe die periphere Durchblutung, Motorik und Sensibilität zu überprüfen und darauf zu achten, dass keine iatrogenen Druckstellen und Hautverletzungen entstehen.

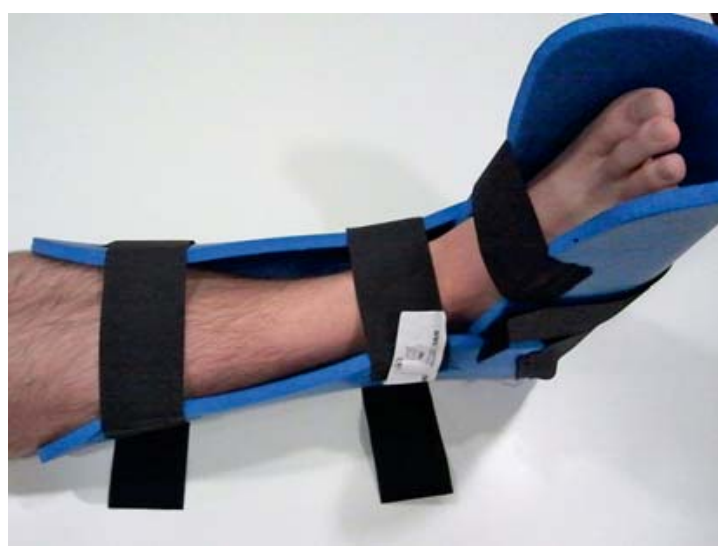

Abb. 4 Sprunggelenkschienung mit Kunststoffsplint.

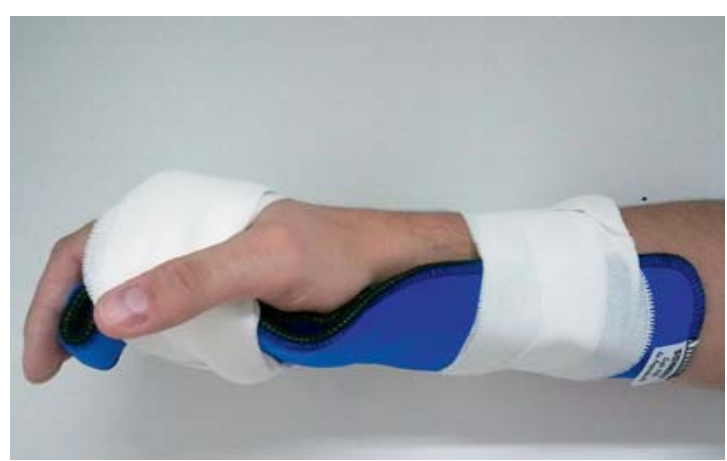

Abb. 5 Handgelenkschienung mit Alupolsterschiene.

\section{Praxis}

Tipps für die Praxis

Zur Lagerung verletzter Extremitäten können präklinisch mit genügend Fantasie viele Alltagsgegenstände oder im Rettungswagen mitgeführte Gegenstände „zweckentfremdet“ werden.

- So können beispielsweise Decken oder Taschen unter die Knie gelegt werden, um eine Anwinkelung der Beine bei einer Hüftluxation zu erreichen.

- Mit Pflasterstreifen kann man verletzte Finger an die danebenliegenden Finger kleben, um sie damit zu schienen. Hierbei sollte allerdings auf die Haut geachtet und gegebenenfalls eine Kompresse dazwischen gelegt werden. 


\section{Kernaussagen}

Grundlagen. Die Behandlung lebensbedrohlicher Verletzungen und der Transport in eine geeignete Zielklinik dürfen durch die Versorgung von nicht lebensbedrohlichen Verletzungen nicht verzögert werden.

Eine möglichst exakte Ersteinschätzung und Beschreibung des Weichteilschadens ist wichtig für die Weiterbehandlung.

Präklinische Diagnostik. Neben Anamnese und klinischer Untersuchung mit der Suche nach sicheren Frakturzeichen sind eine Kontrolle und Dokumentation der peripheren Durchblutung, Motorik und Sensibilität erforderlich.

Geschlossene, nicht dislozierte Frakturen. Der Verdacht auf eine Fraktur genügt, um eine Schienung der Extremität zu initiieren.

Dislozierte Frakturen. Grob dislozierte Frakturen sollen besonders bei Beeinträchtigung der pDMS (periphere Durchblutung, Motorik und Sensibilität) durch dosierten Längszug in Achsrichtung der Extremität reponiert bzw. achsgerecht gelagert werden. Auf entsprechende Analgesie ist zu achten.

Offene Frakturen. Bei offenen Frakturen steht neben der Blutungskontrolle und der Vermeidung weiterer Weichteilschäden durch schonende Reposition und Schienung die Vermeidung von Infektionen im Vordergrund. Hierzu werden grobe Verschmutzungen und Fremdkörper entfernt und nach Reposition ein steriler Verband angelegt.

Amputationen. Das Amputat muss steril verpackt und gekühlt mitgenommen werden.

Blutstillung. Die erste Maßnahme zur Blutstillung ist die manuelle Kompression.

Luxationen. Insbesondere bei gestörter pDMS sollte am Unfallort ein schonender Repositionsversuch durch Längszug unter Analgesie unternommen werden.

Lagerungshilfen. Bei allen Lagerungshilfen ist auf die pDMS und auf mögliche iatrogen gesetzte Druckstellen und Weichteilverletzungen zu achten.

\section{Über die Autoren}

\section{Bernd Wohlrath}

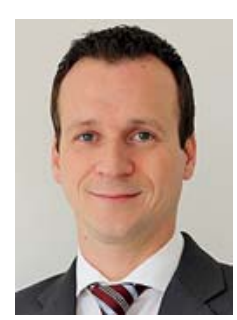

Dr. med. Assistenzarzt der Abteilung für Unfallchirurgie und Orthopädische Chirurgie der BG-Unfallklinik Frankfurt am Main. Notarzt auf dem NEF1 und RTH Christoph 2 (BG-Unfallklinik Frankfurt am Main).

\section{Uwe Schweigkofler}

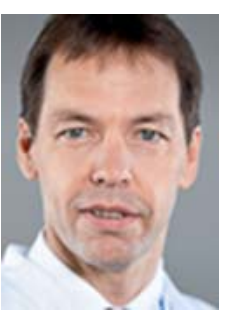

Dr. med. Leitender Arzt des Notfall- und Rettungszentrums der BG-Unfallklinik Frankfurt am Main. Ärztlicher Leiter des NEF1 und RTH Christoph 2 (BG-Unfallklinik Frankfurt am Main). Leitender Notarzt der Stadt Frankfurt am Main.

\section{Reinhard Hoffmann}

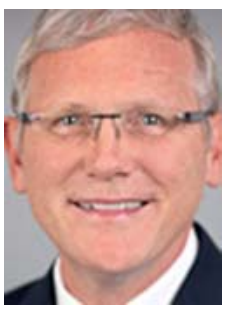

Prof. Dr. med. Ärztlicher Geschäftsführer, Ärztlicher Direktor und Chefarzt der Abteilung für Unfallchirurgie und Orthopädische Chirurgie der BG-Unfallklinik Frankfurt am Main. Generalsekretär DGU. Stv. Generalsekretär DGOU.

\section{Korrespondenzadresse}

Dr. med. Bernd Wohlrath

Abteilung für Unfallchirurgie und Orthopädische Chirurgie Berufsgenossenschaftliche Unfallklinik Frankfurt am Main

Friedberger Landstraße 430

60389 Frankfurt am Main

E-Mail: bernd.wohlrath@bgu-frankfurt.de

Interessenkonflikt: Die Autoren bestätigen, dass kein Interessenkonflikt vorliegt. 


\section{Literatur}

1 TraumaRegister der DGU. Jahresbericht 2014; Sektion NIS der Deutschen Gesellschaft für Unfallchirurgie (DGU)/AUC - Akademie der Unfallchirurgie GmbH. Im Internet: www.traumaregister.de/images/stories/downloads/jahresberichte/TR-DGU-Jahresbericht_2014.pdf; Stand: 02.03.2015

2 Gesundheitsberichterstattung des Bundes. Diagnosedaten der Krankenhäuser ab 2000. Im Internet: http://www.gbe-bund.de/ oowa921-install/servlet/oowa/aw92/dboowasys921.xwdevkit/ xwd_init?gbe.isgbetol/xs_start_neu/

\&p_aid=i\&p_aid=55497787\& nummer=703\&p_sprache=D\&p_ indsp=99999999\&p_aid=76450147; Stand: 27.08.2014

3 NAEMT, Hrsg. Präklinisches Traumamanagement, Prehospital Trauma Life Support (PHTLS). 2. Aufl. München: Elsevier; 2012

4 American College of Surgeons. Advanced Trauma Life Support. ATLS student course manual. 9th ed. Chicago: ACS; 2012

5 Rüter A, Trentz O, Wagner M. Unfallchirurgie. München: Urban \& Schwarzenberg; 2003

6 Tscherne H, Oestern HJ. Die Klassifikation des Weichteilschadens bei offenen und geschlossenen Frakturen. Unfallheilkunde 1982; 85: $111-115$

7 Gustilo RB, Mendoza RM. Problems in the management of type III open fracture: A new classification of type III open fractures. J Trauma 1984; 24: $742-746$

8 Lee C, Porter KM. Prehospital management of lower limb fractures. Emerg Med J 2005; 22: 660-663

9 Kleber C, Lindner T, Bail HJ. Erstversorgung von Frakturen und Luxationen. Notfall Rettungsmed 2009; 12: 551-560
10 Bücking B, Debus F, Ruchholtz S. Präklinische Versorgung von Extremitäten- und Wirbelsäulenverletzungen. Notfallmed up2date 2012: 7: 283-295

11 Cuske J. The lost art of splinting. How to properly immobilize extremities \& manage pain. JEMS 2008; 33: 50-64; quiz 66

12 Deutsche Gesellschaft für Unfallchirurgie - DGU. S3-Leitlinie Polytrauma/Schwerverletzten-Behandlung 2011; AWMF-online Register Nr. 012-019. Im Internet: http://www.awmf.org/leitlinien/detail/I/012-019.html; Stand: 27.08.2014

13 Beck A. Notärztliche Versorgung des Traumapatienten. Notfall Rettungsmed 2002; 1: 57-61

14 Anonymous. Limb trauma. In: School WM, ed. Clinical practice guidelines for use in U.K. Ambulance services. Guidelines of the Joint Royal Colleges Ambulance Liaison Committee and The Ambulance Service Association. London: Warwick Medical School. Im Internet: [http://www2.warwick.ac.uk/fac/med/research/hsri/ emergencycare/guidelines/limb_trauma_2006.pdf; Stand: 27.08.2014

15 Quinn Rh, Macias DJ. The management of open fractures. Wilderness Environ Med 2006; 17: 41-48

16 Lackner CK, Lewan U, Deiler S et al. Präklinische Akutversorgung von Amputationsverletzungen. Notfall Rettungsmed 1999; 2: $188-192$

17 Lee C, Porter Km, Hodgetts TJ. Tourniquet use in the civilian prehospital setting. Emerg Med J 2007; 24: 584-587

18 Beck A. Wunde - Fraktur - Luxation. Notfall Rettungsmed 2002; 8: $613-624$

19 Melamed E, Blumenfeld A, Kalmovich B et al. Prehospital care of orthopaedic injuries. Prehosp Disaster Med 2007; 22: 22 -25

20 Kulla M, Hinck D, Bernhard M et al. Prähospitale Therapiestrategien für traumaassoziierte, kritische Blutungen. Z Notfall Rettungsmed 2014; 17: 575-583 


\section{CME-Fragen}

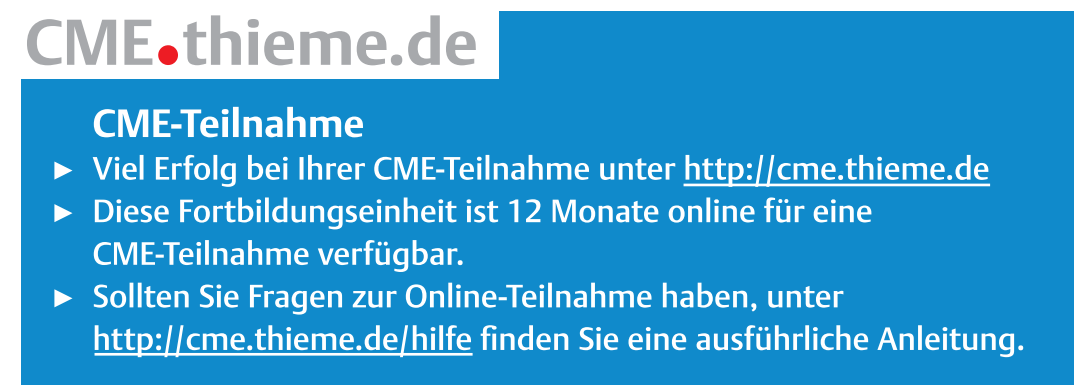

\section{1}

Wodurch ist die Erstuntersuchung Schwerverletzter gekennzeichnet?
A Man beginnt mit einer körperlichen Untersuchung von Kopf bis Fuß.
B Eine Kreislaufkontrolle ist die erste Maßnahme.
C Die Unfallkinematik lässt keine Rückschlüsse auf das Verletzungsmuster zu.
D Die Erstuntersuchung erfolgt nach dem ABCDE-Schema.
E Die Kontrolle der Pupillen ist beim Schädel-Hirn-Trauma die erste Maßnahme.

\section{2}

Eine der folgenden Aussagen zu Extremitätenverletzungen ist falsch. Welche?
A Bei Femurfrakturen können lebensbedrohliche Blutungen nach innen auftreten.

B Bei geschlossenen Frakturen treten keine Weichteilschäden auf.

C Bei Beckenfrakturen können lebensbedrohliche Blutungen nach innen auftreten.

D Die initiale Klassifikation des Weichteilschadens ist wichtig für die Weiterbehandlung.

E Bei schweren und lebensbedrohlichen Verletzungen darf der Secondary Survey den Abtransport in die geeignete Klinik nicht verzögern.

\section{3}

Welcher Befund zählt als sicheres Frakturzeichen?
A Schmerzen
B Schwellung
C Krepitation
D freiliegender Knochen
E Funktionseinschränkung

\section{4}

\section{Welche Aussage zur} geschlossenen Extremitätenfraktur ist richtig?
A Der Verdacht auf eine Fraktur ist Indikation zur Schienung.
B Wenn keine sicheren Frakturzeichen vorliegen, sollte nicht geschient werden.
C Geschiente Beine werden senkrecht nach unten hängend gelagert.
D Die Schienung erfolgt direkt über der Fraktur, ohne die direkt benachbarten Gelenke einzubeziehen.
E Ketten und Ringe an der betroffenen Extremität können bis in die Klinik belassen werden.

\section{5}

Welche Aussage zu dislozierten Frakturen ist falsch?
A Dislozierte Frakturen mit Beeinträchtigung der peripheren Durchblutung sollten reponiert werden.

B Ziel der Reposition ist die achsgerechte Stellung und Wiederherstellung der peripheren Durchblutung.

C Die Frakturreposition ist sehr schmerzhaft.

D Nach erfolgter Reposition sollte die Extremität geschient werden.

E Ist die pDMS vor Reposition intakt, muss sie danach nicht mehr untersucht werden. 


\section{CME-Fragen}

Präklinische Versorgung von Extremitätenfrakturen und Luxationen

\section{6}

Eine der folgenden Aussagen

ist unzutreffend. Welche?
A Bei offenen Frakturen stehen die Blutungskontrolle und die Infektvermeidung im Vordergrund.

B Amputate sollten immer vollständig mitgenommen werden.

C Das Amputat wird direkt auf Eis gelegt und mitgeführt.

D Bei offenen Frakturen kann eine präklinische Antibiotikagabe indiziert sein.

E Offene Wunden werden steril abgedeckt.

\section{7}

Nur eine der folgenden Aussagen ist richtig. Welche?
A Offene Extremitätenfrakturen müssen nicht geschient werden.
B Sterile Verbände auf offenen Frakturen sollten, wenn möglich, erst im OP wieder geöffnet werden.
C Offene Wunden werden präklinisch mit alkoholischer Desinfektionslösung gespült.
D Ziel der präklinischen Frakturreposition ist die anatomische Stellung.
E Die Lokalisation einer Amputation hat keine Auswirkung auf die Auswahl der Zielklinik.

\section{8}

Was ist die erste Maßnahme zur Blutstillung einer einzelnen Wunde?
A Tourniquet anlegen
B Arterie abklemmen
C Arterie abdrücken
D manuelle Kompression
E Druckverband anlegen

9

Welche Aussage ist richtig?
A Bei Weichteilkompromittierungen durch eine Luxation sollte diese zügig reponiert werden.
B Gelenkluxationen sind in der Regel nicht sehr schmerzhaft.
C Eine Schulterluxation muss bei intakter pDMS sofort reponiert werden.
D Die Entscheidung zur Reposition ist unabhängig von der Erfahrung des Arztes.
E Fingergelenkluxationen verursachen keine Weichteilschäden.

\section{0}

Eine der folgenden Aussagen ist unzutreffend. Welche?
A Das Dreiecktuch wird hauptsächlich an den Armen benutzt.
B Die Luftkammerschiene kann Weichteilschäden provozieren.
C Nach der Schienung muss die pDMS kontrolliert werden.
D Die Vakuumschiene kann Weichteilschäden provozieren.
E Die Vakuummatratze eignet sich besonders zur Schienung isolierter Unterarmverletzungen. 